\title{
Protein detection in blood via a chimeric aptafluorescence assay: toward point-of-care diagnostic devices
}

\author{
Andrea Montero-Oleas \\ Cesar Costa-Vera \\ Elizabeth Samaniego Onofre \\ Miguel Angel Mendez
}




\title{
Protein detection in blood via a chimeric aptafluorescence assay: toward point-of-care diagnostic devices
}

\author{
Andrea Montero-Oleas, ${ }^{\text {a,b,c }}$ Cesar Costa-Vera, ${ }^{b}$ Elizabeth Samaniego Onofre, ${ }^{\text {a,b }}$ and Miguel Angel Mendez ${ }^{a, d, e, *}$ \\ anniversidad San Francisco de Quito, Instituto de Simulacion Computacional (ISC-USFQ), Cumbaya, Ecuador \\ bescuela Politecnica Nacional, MSOS Group, Physics Department, Quito, Ecuador \\ 'Universidad San Francisco de Quito, Colegio de Ciencias Biológicas y Ambientales, Laboratorio de Biotecnología Vegetal, Cumbaya, Ecuador \\ 'Universidad San Francisco de Quito, Colegio de Ciencias de la Salud, Escuela de Medicina, Cumbaya, Ecuador \\ eUniversidad San Francisco de Quito, Colegio de Ciencias e Ingenierías Politécnico, Departamento de Ingeniería Química, Grupo de Química \\ Computacional y Teórica (QCT-USFQ), Cumbaya, Ecuador
}

\begin{abstract}
Paper-based analytics allows building portable and disposable devices for point-of-care (POC) diagnosis. Conventional methods for quantifying proteins exhibit substantial disadvantages related to costs and difficulty of the technique when used in settings where fast and cost-effective assays are needed. We report the successful application of a simple, rapid, easy to use, and label-free aptasensor strategy based on the selective fluorescence of the NMM IX dye. For the probe design, the three-dimensional (3-D) structures of the DNA components were carefully analyzed using software for the 3-D visualization of crystallographic structures. The chimeric aptafluorescence molecule consists of two modules, a detection aptamer and a transduction sequence that induces the specific fluorescence of NMM IX. In the presence of thrombin, a fluorescent spot visible to the naked eye can be observed. The fluorescent response is directly proportional to protein concentration and can be easily quantified colorimetrically using a low-cost microscopy system. The recognition probe design might be adaptable to other relevant biological analytes by changing the sequence of the aptamer. This proof of principle represents a contribution to the development of useful, cheap, reliable, and simple protein quantification assays for POC testing. () 2018 Society of Photo-Optical Instrumentation Engineers (SPIE) [DOI: 10.1117/1.JBO.23.9 .097003]
\end{abstract}

Keywords: aptasensor; in silico design; label-free; N-methyl-mesoporphyrin IX; point-of-care device; chimeric aptafluorescence assay.

Paper 180321R received Jun. 2, 2018; accepted for publication Sep. 4, 2018; published online Sep. 25, 2018.

\section{Introduction}

Detection and quantification of proteins are crucial in many fields of science, principally in the development of molecular diagnostic tools. ${ }^{1}$ For this reason, the implementation of simple, rapid, and sensible methods is essential in the clinical medicine field. ${ }^{2}$ Conventional protein quantification methods are highly sensitive and selective; however, they are expensive and require trained personnel, expensive instrumentation, and laborious pretreatments of the samples. $^{3-5}$ Lately, point-of-care (POC) diagnostics have been widely studied as an alternative for resource-limited settings, necessary in developing countries. ${ }^{6}$ POC devices consist of rapid, portable, and easy to use biosensors for the detection of biological samples associated with a disease or health condition. 6,7

Aptasensors are playing an essential role in the development of biosensors. Aptasensors use aptamers as a biological sensor for molecular recognition with high affinity and specificity. ${ }^{8-10}$ The aptasensor reporter system consists of an aptamer probe and a reporter molecule, such as a fluorophore or a dye molecule. These reporters can be labeled or label-free. ${ }^{11}$ A labeled reporter system consists of an aptamer chemically bound to the reporter molecule. ${ }^{12}$ In contrast, in label-free systems, reporters are free

*Address all correspondence to: Miguel Angel Mendez, E-mail: mmendez@ usfq.edu.ec fluorophores or dyes that have a particular specificity with the aptamer structure or sequence. ${ }^{13}$

DNA fluorescent dyes with interaction-responsive emission have been successfully used in the development of labelfree fluorescent aptasensors. ${ }^{14}$ These dyes allow the development of cheaper, versatile, and straightforward fluorescence biosensors. ${ }^{15}$ N-Methyl-mesoporphyrin IX (NMM IX) is an anionic porphyrin, characterized by a remarkable structural selectivity for parallel G-quadruplexes DNA. ${ }^{16}$ The fluorescence of NMM IX is very weak but exhibits a $>20$-fold enhancement upon binding to a parallel G-quadruplex DNA. Due to this feature, various authors have used NMM IX and DNA for the detection of a variety of targets like proteins, lead ions, oligonucleotides, and different types of nanoparticles. ${ }^{17-20}$

Thrombin binding aptamers (TBAs) are the most commonly used model systems to demonstrate the proof of concept of aptasensors assays for several reasons. First, usually TBAs are short oligonucleotides; hence, are easy and inexpensive to synthesize. ${ }^{21}$ Second, the dissociation constants of the best characterized TBAs are small, in the nanomolar range; therefore, the affinity between the aptamer and the thrombin is high. ${ }^{21,22}$ Also, thrombin is a blood protein, which concentration may vary from $\mathrm{pM}$ to $\mu \mathrm{M}$ levels, and consequently, thrombin aptasensors have low enough detection limits as to permit validation at the nanomolar range detection for a POC assay. ${ }^{23,24}$

$1083-3668 / 2018 / \$ 25.00$ @ 2018 SPIE 
Herein, we report the development of a simple, rapid, easy to operate, label-free chimeric aptafluorescence assay (CAFA) for thrombin quantification in blood samples. Our CAFA is based on the use of a thrombin recognition probe, which consists of two oligonucleotide modules built into one construct that can be named as chimeric probe. The first module is a TBA, and the second module is a DNA tertiary structure that binds specifically to NMM IX. In this work, we report the successful application of this simple assay to the identification of thrombin in blood, by measuring the fluorescence emitted by the aptafluorescence probe (AFP) formed by NMM IX and the chimeric probe. The colorimetric response was visible to the naked eye, and we implemented a low-cost microscopy system for quantitative analysis of the stains. Beforehand, to design the chimeric probe, we employed in silico molecular tools. These tools aided in the selection of the most suitable DNA sequence needed for the protein recognition in blood. Furthermore, these tools could potentially be used for the design of a wide range of recognition molecules for the quantification of other blood proteins. This study represents the initial phase in the development of a useful, cheap, reliable, and simple protein quantification method with potential to be translated into a POC device.

\section{Materials and Methods}

\subsection{Materials and Reagents}

All deoxyoligonucleotides were purchased from Midland Oligos (Midland, Texas). The sequences, names, and abbreviations used in this work for each oligonucleotide are shown in Table 1. Thrombin from human plasma (lyophilized powder, $\geq 1000 \mathrm{NIH}$ units/mg protein) was supplied by Sigma-Aldrich (St. Louis, Missouri). Cellulose nitrate white sterile membrane filters $3-\mu \mathrm{m}$ pore size were purchased from Sartorius (Göttingen, Germany). N-Methyl-mesoporphyrin IX was ordered from Frontier Scientific (Sunnyvale, California). Human plasma samples were obtained from volunteer donors. The acquisition of the samples and their use was performed in compliance with relevant laws and institutional guidelines and oversaw by a university bioethics committee (Comité de Ética de Investigación en Seres Humanos, Universidad San Francisco de Quito). Thrombin and the NMM IX dye were dissolved in ultrapure distilled water. Oligonucleotides were resuspended in TE $1 \mathrm{X}$ buffer (5 mM Tris-HCl, $0.5 \mathrm{mM}$ EDTA, $\mathrm{pH} 7.0$ ) and quantified in a Nanodrop 1000 (ThermoScientific, Waltham, Massachusetts). For the folding of the chimeric probe, the oligonucleotide suspensions were heated at $95^{\circ} \mathrm{C}$ for $10 \mathrm{~min}$ and subsequently mixed with an equal volume of 2X HEPES buffer [50 mM HEPES, $40 \mathrm{mM} \mathrm{KCl,} 400 \mathrm{mM}$ $\mathrm{NaCl}, 0.1 \%$ (w/v) TritonX-100, $2 \%$ (v/v) DMSO, pH 7.0]. Then, the solutions were heated to $95^{\circ} \mathrm{C}$ for $10 \mathrm{~min}$ before let to cool slowly to $20^{\circ} \mathrm{C}$ to allow assembly. Cations other than $\mathrm{Na}^{+}$and $\mathrm{K}^{+}$were carefully avoided in the final buffer solutions to prevent undesired effects over the oligonucleotide folding processes.

\subsection{Sequence and Three-Dimensional Structure Analysis for the Design of the Chimeric Probe}

The first step for the chimeric probe design was to identify all available 3-D structures for (i) DNA aptamers that specifically may bind to thrombin (i.e., HD1 aptamer) and (ii) parallel G-quadruplex DNA structures that could trigger the fluorescence of NMM IX (i.e., c-Myc). Previously, we have reported systems where the same DNA molecule folded on a G-quadruplex structure that exhibits medium fluorescence intensity by itself in certain cases. ${ }^{25}$ In addition, G-quadruplex structures can bind to small organic dyes with good specificities. ${ }^{25,26}$ Furthermore, the NMM IX dye fluoresces with high intensity when bound to parallel G-quadruplexes but does not fluoresce or fluoresces with much lower intensity, when bound to other DNA structures. ${ }^{16}$ These considerations were critical for the selection of the chimeric probe as the reporter for our study.

As a second step, we performed an in silico 3-D structure analysis where both nucleotide components were visualized with VMD (University of Illinois at Urbana-Champaign, version 1.9.2) to define molecular structure-based criteria to design a single DNA strand sequence that when folded will contain the two main elements - the aptamer and the parallel G-quadruplex oligo-without interfering with each other. Visualizing the structures, we identified intramolecular interactions directly involved in the assembly of each molecule in the chimeric probe. An important question for the chimeric probe construction is whether or not to use a linker sequence between the building blocks to avoid unwanted DNA structures. We investigated the question by checking if when the two sequences bind, there was not a section with a prima facie substantial probability to form other tertiary superstructures. For this, we considered that if the part of the whole sequence that acts as a bridge has too many guanine residues, it could lead to the formation of undesired G-quadruplex structures. To solve that problem, we count the number of available guanines in the space between the blocks. Based on this analysis, we

Table 1 Abbreviations, descriptive labels, and sequence of the deoxioligonucleotides.

\begin{tabular}{llll} 
Abbreviation & \multicolumn{1}{c}{ Sequence description } & \multicolumn{1}{c}{ Descriptive label } & \multicolumn{1}{c}{ Sequence 5' $\rightarrow 3^{\prime}$} \\
\hline- & Designed chimeric probe & Chimeric probe & GGTTGGTGTGGTTGGTTTAAGGGTGGGTAGGGTGGGTAA \\
HD1 & TBA & HD1 aptamer & GGTTGGTGTGGTTGG \\
C-MYC & Parallel G-quadruplex oligo & c-Myc & TGAGGGTGGGTAGGGTGGGTAA \\
NACP & Non assembled chimeric probe & Chimeric probe & a \\
MCP & Mutated chimeric probe control & Mutated chimeric probe & GGTTGGTGTGGTTGGTTTAATTTTGGGTAGGGTGGGTAA \\
\hline
\end{tabular}

Note: The mutations are indicated in bold. The thymine linker is shown in italics.

${ }^{a}$ The difference with the chimeric probe is that the 3-D assembly is not performed. 
concluded that a linker is needed to provide the desired 3-D structure.

Independently of the previous analysis, the protein structure and the binding sites of blood DNAses were inspected to make sure that they will not cleave, or else strongly modify the chimeric probe when implementing the biosensor for blood samples. If the 3-D structure was available, we visualized it with VMD, and identified the type of 3-D structure of the DNA ligand that binds to the enzyme. If the 3-D structure was not available, we checked the literature for other evidence. ${ }^{27-31}$

To verify if the in silico chimeric probe design was adequate, we tested control sequences via both CAFA and, absorbance and emission spectroscopy validations. Controls for the spectroscopy validation were a TBA and a parallel G-quadruplex oligonucleotide. For the CAFA, we tested the same controls used in the spectroscopic analysis plus two controls that consisted of the chimeric probe designed with suitable modifications. One control had the same sequence as the designed chimeric probe; the difference was that the assembly process described in Sec. 2.1 was not performed before carrying out the CAFA. The second control probe was the same chimeric probe with the difference that we substituted three Gs for three Ts (residues 21, 22, and 23) and other G for A (residue 19) in the parallel G-quadruplex oligonucleotide as shown in Table 1.

\subsection{Absorbance and Emission Spectroscopy from Solution Samples}

Spectroscopic methods were used to characterize the correct folding of the construct and its potential use as a reporter molecule both in solution, and on the surface of a nitrocellulose membrane. For this, we used a 215- to 2500-nm light source (DT-Mini, Ocean Optics, Dunedin, Florida) to measure in solution the absorbance spectra, and a 405-nm excitation diode laser to excite fluorescence. The corresponding spectra of the chimeric probe and two controls were measured. The controls were, on one hand, a solution of a parallel G-quadruplex DNA sequence and, on the other hand, a solution of the TBA. For the absorbance and emission spectra acquisition, an aqueous solution of $1 \mu \mathrm{M}$ NMM IX was prepared and used for all measurements. A 10-time excess concentration of the assembled DNA (chimeric probe or control) was added to the NMM IX solution $^{16}$ to verify the successful assembly of the complex by checking if a red shift in the absorbance spectrum was observed in these samples, as explained later.

\subsection{Chimeric Aptafluorescence Assay for Thrombin Detection on the Surface of a Membrane}

Once the chimeric probe was shown to properly assemble, we proceeded to implement a versatile, simple, and economical protocol for thrombin detection using a similar strategy to wellknown blotting techniques. The proposed technique consists in immobilizing the blood plasma sample on the surface of a solid matrix, followed by the addition of the chimeric probe and the NMM IX dye. The chimeric probe acts both as a thrombin detector and as a reporter complex in the presence of the dye. The unbound chimeric probe is removed by washing the matrix several times, thus avoiding unspecific binding and false positives. If there is no thrombin in the sample, the chimeric probe and the NMM IX dye are washed and no fluorescence is observed.

In our design, the nitrocellulose membrane was previously activated by immersion on $1 \times$ TBS buffer. The membrane was allowed to dry for $5 \mathrm{~min}$. The blood samples were collected in sodium citrated anticoagulant tubes and centrifuged at $1500 \mathrm{rpm}$ for $15 \mathrm{~min}$ to recover only blood plasma. About $1 \mu \mathrm{L}$ of plasma aliquots was spotted on the membrane. Next, the membrane was blocked by immersion on 1× TBS buffer for $30 \mathrm{~min}$ and allowed to air dry. From this point, two methodologies were tested to find an optimal protocol for thrombin detection on the blood plasma samples (Fig. 1). In the first methodology (one-step methodology), the AFP was constructed by preincubation of the chimeric probe with the NMM IX dye at room temperature for $1 \mathrm{~h}$. About $2 \mu \mathrm{L}$ of this mixture was spotted on the membrane. On the second methodology (two-steps

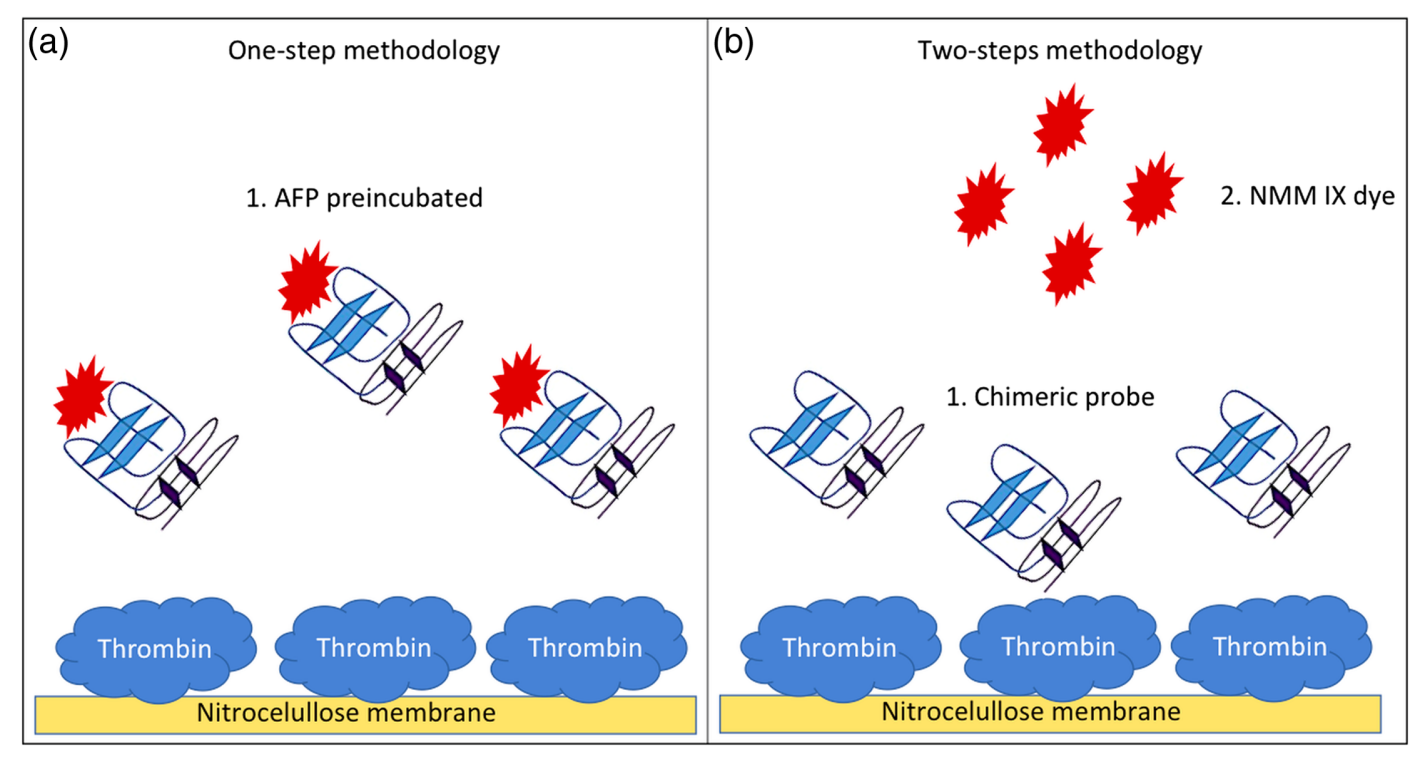

Fig. 1 Methodologies used for the CAFA. (a) One-step methodology, the NMM IX dye, and the chimeric probe were previously mixed forming the aptafluorescence probe (AFP) before the addition to the nitrocellulose membrane. (b) Two-step methodology, the chimeric probe was first mixed with thrombin on the membrane, and in a second step, the NMM IX was added. 
methodology), $2 \mu \mathrm{L}$ of the chimeric probe was added directly to the samples on the membrane and was allowed to react for $1 \mathrm{~h}$ at RT, before rinsing for three times with $1 \times$ TBS buffer with Tween20. Next, $2 \mu \mathrm{L}$ of NMM IX was added to the sample spots (Fig. 1). That is, while in the one-step methodology, the AFP was constructed before its application on the membrane; in the two-step methodology, the chimeric probe is first incubated with the thrombin and then incubated with the dye to form the AFP. Three final rinses with $1 \times$ TBS buffer with Tween 20 were performed to eliminate all the nonspecifically bound molecules of the chimeric probe and the NMM IX dye.

To validate the design of the chimeric probe, we tested control probes with the AFP assay. The control probes, which were previously described in Sec. 2.2, were (1) the TBA, (2) the parallel G-quadruplex oligo, (3) the nonassembled chimeric probe, and (4) the modified chimeric probe control.

Once the proper methodology was selected, we proceeded to optimize the concentration of the chimeric probe and the NMM IX dye. The concentrations tested for the probe and the NMM IX were 10:5, 10:10, 10:20, 20:5, 20:10, 20:20, 40:5, 40:10, and $40: 5 \mu \mathrm{M}$ (chimeric probe:NMM IX dye, respectively); the three immersion times were 2,5 , and $10 \mathrm{~min}$, respectively.

\subsection{Low-Cost Point-of-Care Measurement System}

For the measurement system, we used an optical microscope (Motic, Richmond, British Columbia, Canada), the light of a UV lamp (DT-Mini) pointed via a suitable optical fiber directly at the membrane, and a high-pass filter $(500 \mathrm{~nm}$, Thorlabs, Newton, New Jersey) positioned in between the optical imaging system and a CCD camera (5 Mp, Motic, Richmond, British Columbia, Canada) used to acquire images. Images were analyzed with ImageJ v.1.5. ${ }^{32}$ The intensity of the spots was calculated by measuring the mean gray value, sampling in five equivalent different circular regions in the spot. These circular regions were selected randomly avoiding only the edges of the spots. All measurements were done in triplicate.

\subsection{Standard Addition Technique for Thrombin Quantification}

The standard addition technique was performed to obtain a calibration curve of the image quantification of six thrombin concentrations: $1 \mathrm{nM}, 5 \mathrm{nM}, 50 \mathrm{nM}, 0.5 \mu \mathrm{M}, 5 \mu \mathrm{M}$, and $10 \mu \mathrm{M}$ of thrombin diluted on $1 \%$ plasma samples. All the samples were processed according to the protocol described in Secs. 2.4 and 2.5 .

\subsection{Whole Blood Aptasensor Point-of-Care Assay}

To build a POC device, we tested the CAFA using whole blood as a sample (instead of plasma). Blood samples were collected using Accu-Chek lancets (F. Hoffmann-La Roche AG, Basel, Switzerland), in blood collection tubes both with and without citrate as an anticoagulant. Blood samples were processed, directly or diluted in water, following the protocol described in Secs. 2.4 and 2.5. To find the best test, we used as parameters the intensity of the spot formed and the size of the halo generated in it (plasma halo thickness). The plasma halo thickness was measured in the software ImageJ by tracing a straight line transverse to the halo generated in the spot. The intensity was measured only in the area corresponding to the generated halo.

\subsection{Statistical Evaluation}

A Shapiro-Wilk test was performed to analyze whether the measurements are normally distributed or not. In all the samples, the null hypothesis was rejected ( $p>0.05$ ), hence an ANOVA oneway test was performed and subsequently a Tukey test to identify significant differences between each assay.

\section{Results and Discussion}

\subsection{Sequence and Three-Dimensional Structure Analysis for the AFP Design}

The technological advances allow the use of sophisticated and easy to use computational tools that can contribute significantly to speed up the experimental design. Without a doubt, software for molecular visualization is a crucial tool for the evaluation of complex molecular recognition assemblies. ${ }^{33,34}$ We demonstrate in this work evidence of these capabilities by constructing an aptamer-based biosensor for thrombin detection in blood plasma samples and whole blood samples. With the use of in silico molecular visualization tools, we avoided unnecessary trials and error experiments in the selection of detection probes for the biosensor, saving time, and resources.

Based on the sequence and 3-D structure analysis of candidate oligonucleotides reported in the literature, the selected sequences to build the chimeric probe were the TBA HD $1^{35-37}$ and the c-Myc oligonucleotide (the parallel G-quadruplex structure).$^{38-40}$ In the literature, six TBAs were found as possible candidates to form the chimeric probe. Aptamer HD1 was selected because it is the best characterized, has the most straightforward structure, and the shortest sequence. Moreover, for the selection of the parallel G-quadruplex oligonucleotide, four candidates were analyzed. From these, the oligonucleotide c-Myc was selected because it was the only one with a unimolecular structure. It has also been reported in the literature that in the presence of the c-Myc G-quadruplex structure, NMM IX dye fluoresces higher than in the presence of the other oligonucleotides. ${ }^{16}$

For the chimeric probe construction, two possible arrangements of the selected oligonucleotides were considered plausible (5'-c-Myc/HD1-3' and 5'-HD1/c-Myc-3'). The c-Myc/ HD1 arrangement was discarded as it was observed that the thrombin binding site in HD1 is too close to the 5'end, so the assembly of c-Myc might potentially block this site. The HD1/c-Myc arrangement was selected upon the introduction of proper modifications in its sequence. These modifications were meant under consideration that the union of both oligonucleotides leaves three consecutive guanines, which can generate the formation of an undesired G-quadruplex structure and modify the original 3-D structure of the sequences. Based on the a priori 3-D evidence mentioned in Sec. 2.2, a linker consisting of two thymines was introduced between the sequences of these two blocks to avoid the formation of undesirable structures. Additionally, a substitution (G/A) on the original sequence of c-Myc was needed to prevent the formation of unwanted tertiary structures. In this manner, two thymines were attached to the 3'end guanine of the HD1 aptamer (residue 15 ), and this framework was attached to the 5'end thymine of the modified C-Myc oligonucleotide (residue 1) (Fig. 2). 


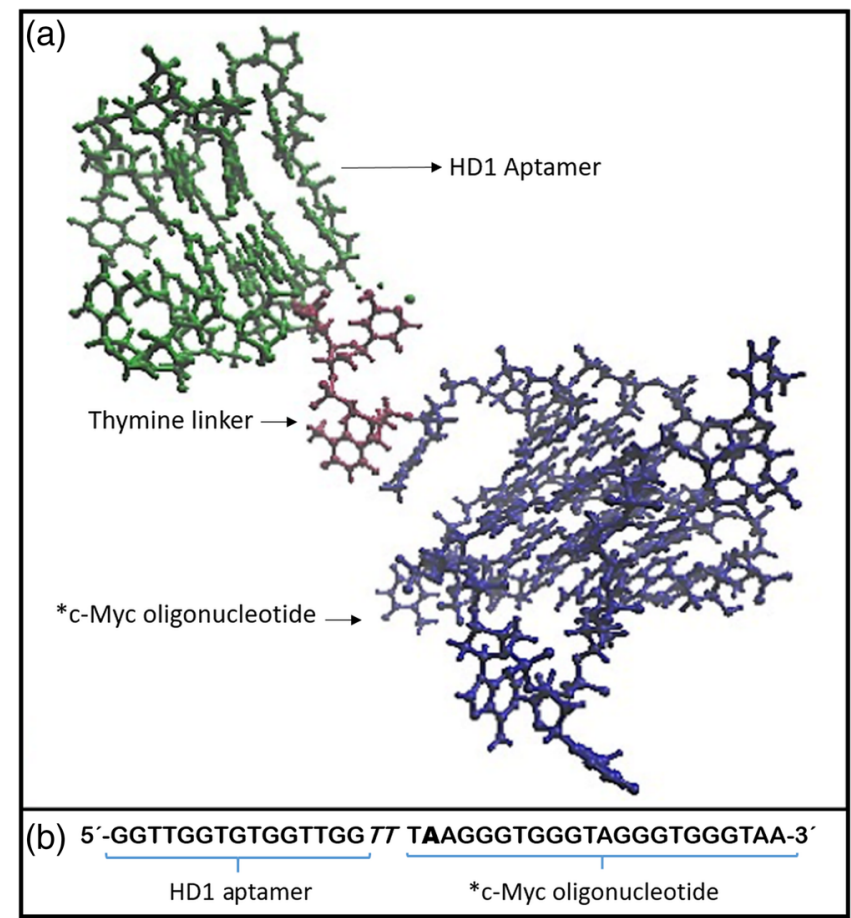

Fig. 2 Chimeric probe selected for the CAFA for thrombin detection. (a) Ball-and-stick visualization of the chimeric probe. (b) Chimeric probe sequence. It can be observed the linker of two thymes in italics and in bold the substitution G/A in C-Myc. ${ }^{*} \mathrm{C}-M y c$ sequence modified as described in Sec. 3.1. Image generated based on PDBs 4DIH and 1XAV.

A similar approach could be used to design a CAFA for other relevant molecular targets allowing the implementation of the experimental protocol reported here for many other bioanalytical applications.

\subsection{Spectroscopic Characterization of the Chimeric Probe Assembly in Solution}

When the NMM IX dye is in the presence of a parallel G-quadruplex DNA (c-Myc), two phenomena occur: the emission of the dye increases and the absorption spectra is red-shifted. ${ }^{16,41}$ Both phenomena were observed in the corresponding absorption and emission spectra in solution (Fig. 3). For NMM IX, an absorption peak at $378 \mathrm{~nm}$ [Fig. 3(a)] and a very low fluorescent emission were observed [Fig. 3(b)]. However, as expected, a significant increase in emission occurred when the same dye solution was mixed with the chimeric probe [Fig. 3(b)]. Also, a prominent $21-\mathrm{nm}$ red shift in the absorbance spectrum was observed [Fig. 3(a)]. These two findings demonstrate that in solution, the chimeric probe is folding as expected. ${ }^{16}$ In the case of the control solution NMM IX-HD1, a lower fluorescence than that of the chimeric probe was observed, as also expected. A lower fluorescence is consistent with previous reports where G-quadruplex nonparallel oligonucleotides, like the HD1 aptamer, induced lower fluorescence than parallel G-quadruplex oligonucleotides. ${ }^{16}$ For the NMM IX-c-Myc solution control, the fluorescence was almost as intense as that of the chimeric probe. This suggests that the G-quadruplex parallel structure was conserved when the c-Myc was added as a module within the chimeric probe. In summary, in solution, the chimeric probe shows the same fluorescence than c-Myc control and higher
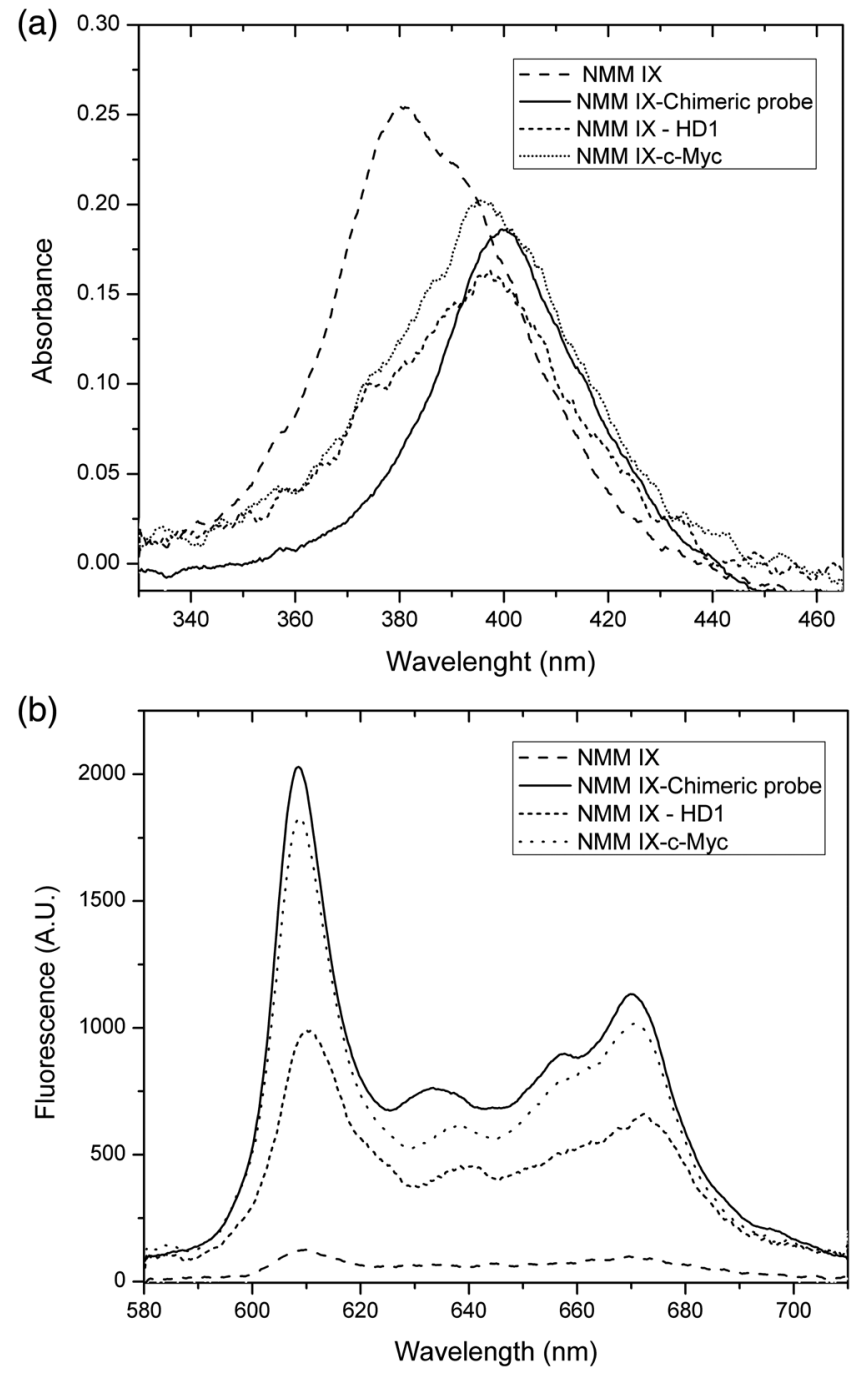

Fig. 3 (a) Absorbance spectra of a solution of NMM IX in the presence of different oligonucleotides. The expected red shift in the absorbance maximum is observed for the NMM IX-chimeric probe. (b) Emission spectra of a solution of NMM IX in the presence of different oligonucleotides. A noticeable increase in emission intensity is appreciable in the NMM IX-chimeric probe solution.

fluorescence than HD1 aptamer and, by these means, demonstrates full detection capabilities.

\subsection{Chimeric Aptafluorescence Assay for Chimeric Probe Design Validation}

The CAFA was carried out for the validation of the designed chimeric probe by means of the detection of thrombin in blood plasma. For this purpose, we used the results obtained with the one-step methodology. The reason for this choice is explained in detail in Sec. 3.4.

The results showed that when we immobilized thrombin on the surface of the nitrocellulose matrix, AFP worked by binding to thrombin in the first place, and then reporting a fluorescent signal that can be easily translated into thrombin abundance. This fluorescence can be easily detected with our low-cost measurement system. In addition, the results of the CAFA performed on the controls also allow validating the performance of the chimeric probe. The CAFA carried out with the HD1 
(a)
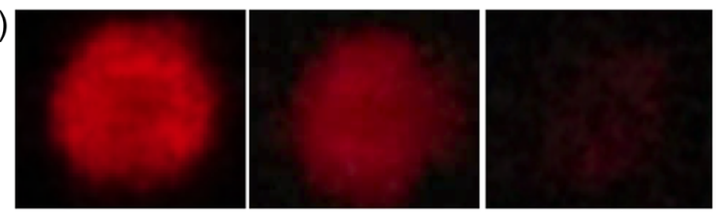

$\mathrm{CP}$

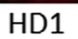

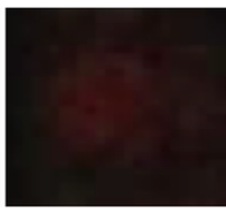

MCP

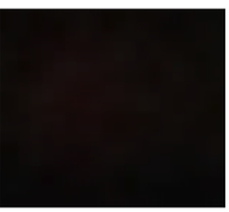

c-Myc

(b)

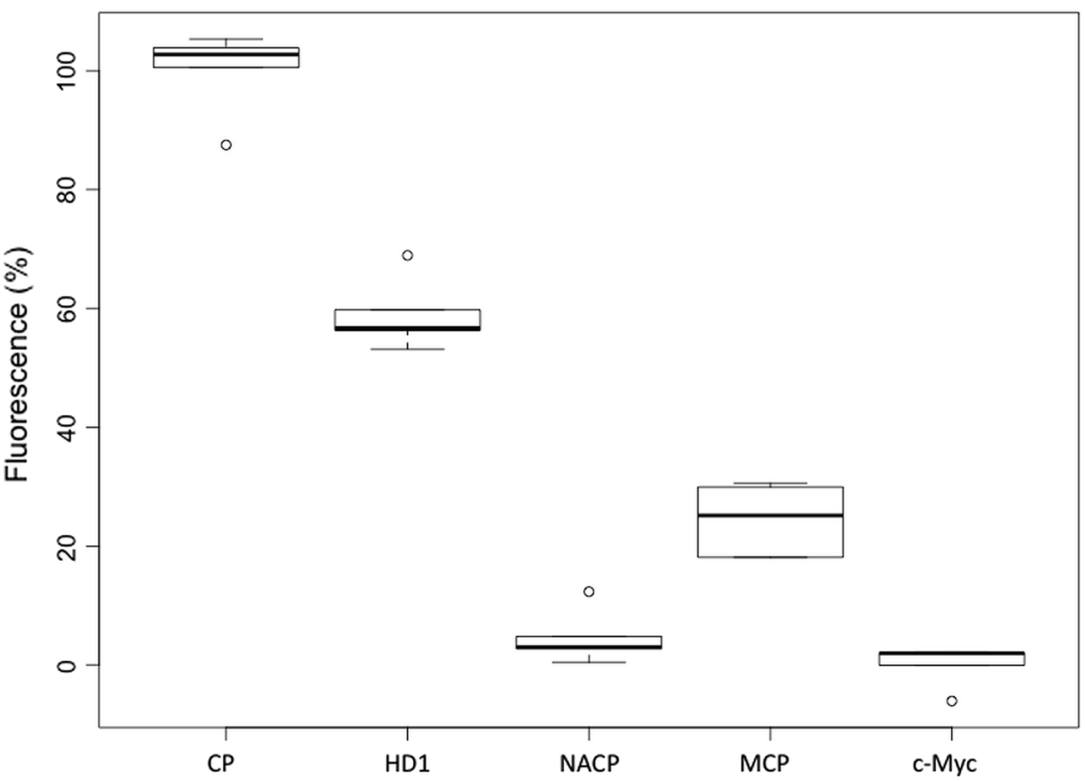

Fig. 4 CAFA performed to validate the chimeric probe design. CP: chimeric probe; HD1: thrombin binding aptamer; NACP: non assembled chimeric probe; MCP: mutated chimeric probe and C-Myc: negative control. (a) Optical microscopy images of the CAFA performed with the chimeric probe designed and different controls. (b) Box plots of the fluorescence signal (normalized to c-Myc assay) for each spot calculated from the microscopy image.

aptamer as probe control, showed a significant fluorescence signal because it has an antiparallel G-quadruplex structure (Fig. 4). It has been reported that NMM IX fluorescence increases 60-fold in the presence of parallel G-quadruplex structures and only 10-fold when it is an antiparallel G-quadruplex. ${ }^{16,17,42}$ Therefore, in the presence of thrombin, the antiparallel HD1 probe attached to the dye binds to the membrane and fluoresces efficiently (Fig. 4); however, the difference is evident when comparing the fluorescence of the chimeric probe treatment versus the HD1 control probe treatment ( $p=10^{-7}$ for the Tukey multiple comparisons of means). The enhanced fluorescence of the chimeric probe in comparison with that of the HD1 probe in CAFA demonstrates that the presence of the c-Myc oligonucleotide is essential to obtain a biosensor with higher sensitivity and accuracy (Fig. 4).

In contrast, the CAFA performed on c-Myc acts as a negative control because it does not bind to the thrombin immobilized in the membrane and washes from the matrix almost entirely. As expected, this results on a very weak signal (Fig. 4).

Moreover, we tested a nonassembled chimeric probe (NACP) and a mutated chimeric probe (MCP) to demonstrate that both the thrombin detection and reporter capability depend on their 3D structure. As expected, the results for both assays resulted again in a weak signal (Fig. 4). With the NACP probe, the signal was comparable to that observed with the c-Myc negative control assay. As the assembly procedure was not performed with NACP, the probe does not bind to thrombin, and therefore, it is removed during the washing. Consequently, does not fluoresce, similarly to what happened with the c-Myc assay (Fig. 4). Also, the MCP probe did not assemble because of the mutation introduced into the c-Myc sequence, and as a consequence, the corresponding observed fluorescence was weaker that the successful probes (Fig. 4). We suspect that the substituted nucleotides in the sequence introduced new structural

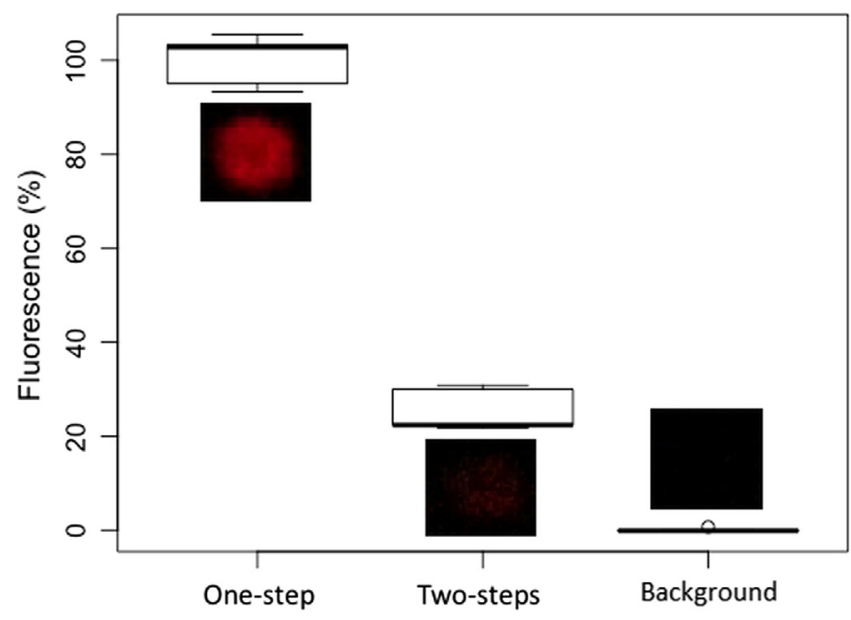

Fig. 5 Comparison of the fluorescence obtained with the CAFA carried out with the one-step methodology and the two-step methodology. Box plots show fluorescence (normalized to the membrane background) for each spot calculated from the optical microscopy image. The one step fluorescence is about four times larger than the best two-step case. 
interactions, forcing the MCP to adopt a nonbinding structure. The results obtained with the NACP and MCP controls show that the computational analysis of the chemical probe structure is very useful to design adequate probes for the CAFA.

\subsection{Optimization of Chimeric Aptafluorescence Assay}

The one-step methodology was found to be the most efficient, as it generated the highest fluorescence signal observed (Fig. 5).
That is, a significantly higher fluorescence signal was produced when the AFP was formed by a probe-dye preincubation (the one-step methodology) in comparison to when the incubation occurred directly on the membrane $(p<0.002$ for the Tukey multiple comparisons of means). In the one-step methodology, the interaction between the probe and the dye occurs in solution, whereas in the two-step methodology, this interaction happens on the surface of a solid matrix. In solution, the available volume is larger, and molecules can arrange themselves to better interact, which does not occur as easily on the solid matrix. The

(a)
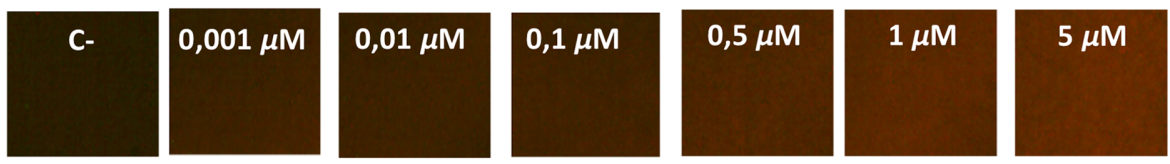

(b)

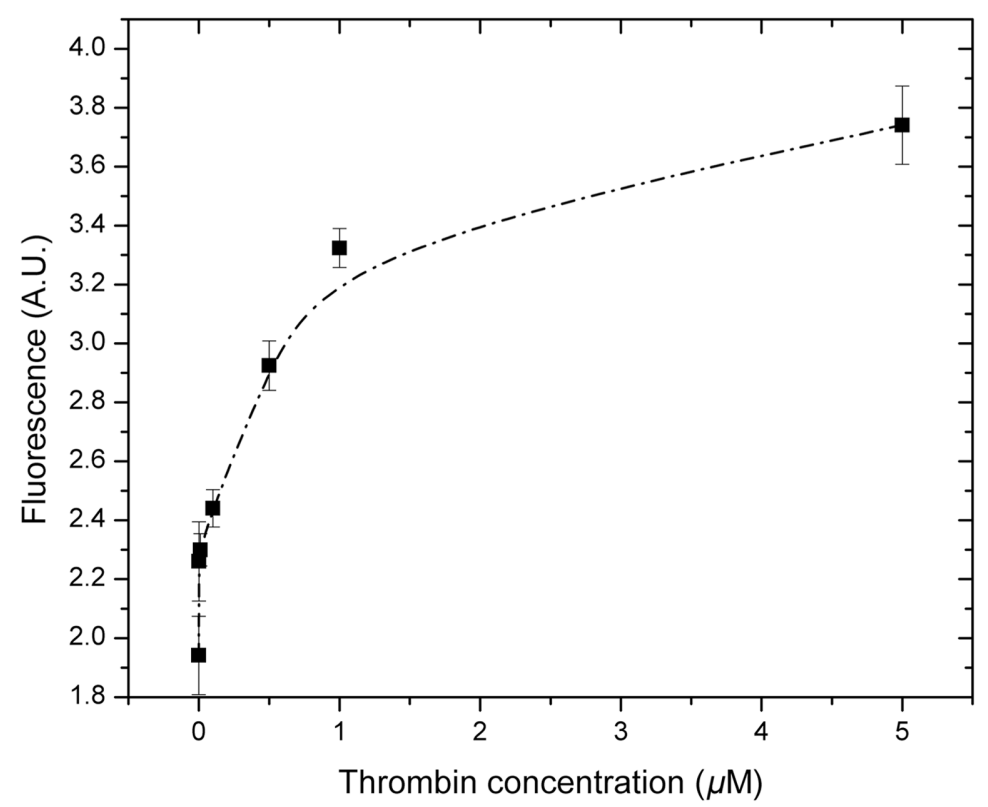

(c)

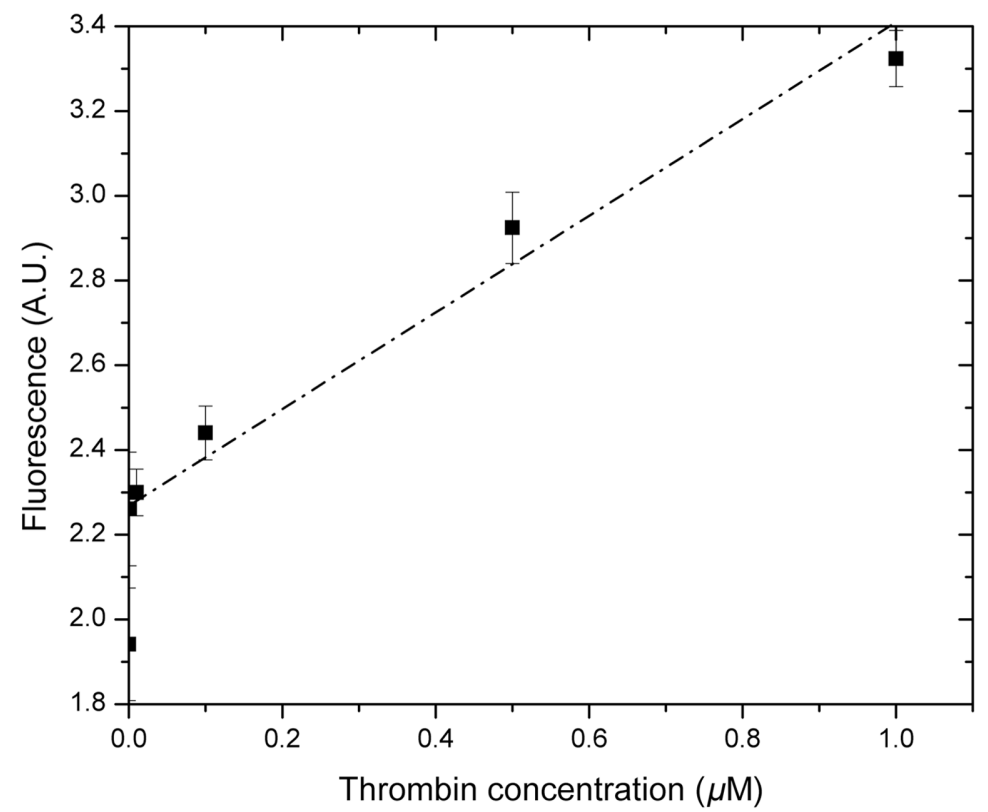

Fig. 6 A. Standard addition technique for quantitative CAFA with different concentrations of thrombin diluted in $1 \%$ blood plasma. (a) Optical microscopy images of the standards. (b) Saturation curve obtained with all the thrombin concentration tested. (c) Linear fit calibration curve for the data in (b) $\left(R^{2}=0.91\right)$. 
result is $\sim 4$ times larger fluorescence signal in the one-step methodology and as a consequence, greater assay sensitivity (Fig. 5).

Regarding the optimization of the ratio between the chimeric probe and the dye concentrations, it was found that the best ratio was $40-\mu \mathrm{M}$ chimeric probe: $20-\mu \mathrm{M}$ NMM IX. Additionally, for the immersion times on the washing buffer, the best conditions were obtained when we performed three immersions of $5 \mathrm{~min}$ each. With this method, there is not a significant difference in signal intensity (with a $p=0.052$ ) between the c-Myc assay and the signal of a spot, where plasma alone was incubated with the dye. Therefore, we conclude that the dye nonspecifically bound to the membrane completely washes away.

\subsection{Chimeric Aptafluorescence Assay-Quantification Assay}

For the experimental conditions on the CAFA-quantification assay, we maintained constant the concentration ratio $(40-\mu \mathrm{M}$ chimeric probe: $20-\mu \mathrm{M}$ NMM IX); therefore, there is a limit in the amount of thrombin that can bind this limited amount of aptamer. As expected, we found that the curve follows a typical saturation behavior (Fig. 6). To quantify the amount of thrombin is more convenient to use only the linear region, and for all the following calculations, we used the section shown in Fig. 6(c). The calibration curve [Fig. 6(c)] obtained by the standard addition technique indicates an almost linear association between the color intensity of the spot and the thrombin concentration in plasma (correlation coefficient $\left.R^{2}=0.91, p=0.0015\right)$. The association found means that the assay successfully permits quantifying thrombin in plasma with a suitable sensitivity.

Furthermore, this behavior can easily be observed with the naked eye. At a $0.001-\mu \mathrm{M}$ thrombin concentration, the color intensity of the spot is very similar to that of the plasma control. The standard definition of detection limit (LOD) establishes that the lowest detectable concentration should be equal to the concentration of sample corresponding to the blank signal value plus three standard deviations of the blank. ${ }^{43}$ By this definition, the LOD of our method is calculated to be $0.11 \mu \mathrm{M}$. Standard additions with higher concentrations to the ones shown in Fig. 6 were not performed, as the typical concentrations of thrombin and other expressed proteins in blood in human blood range between $0.47 \mathrm{mM}$ for albumin and $0.042 \mathrm{nM}$ for Troponin I approximately. ${ }^{44}$

\subsection{Toward a Point-of-Care Diagnostic Device}

The results obtained in the whole blood assay demonstrate that it is not necessary to employ pure plasma in the CAFA. The assay works efficiently using whole blood, and it is not necessary to use any anticoagulant. In this way, this biosensor can be part of a strategy for the development of a POC device.

Figure 7(a) shows the spot obtained when processing a diluted sample of blood with the protocol that we propose. It is observed that the thrombin of the sample is concentrated

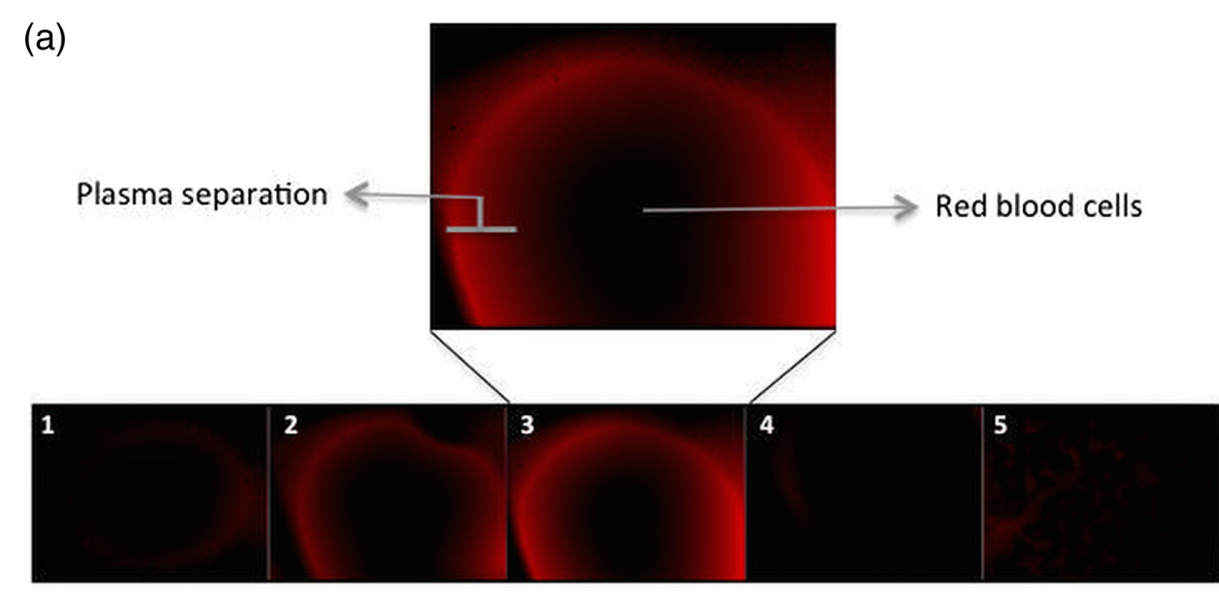

$\square$ Intensity (A.U)

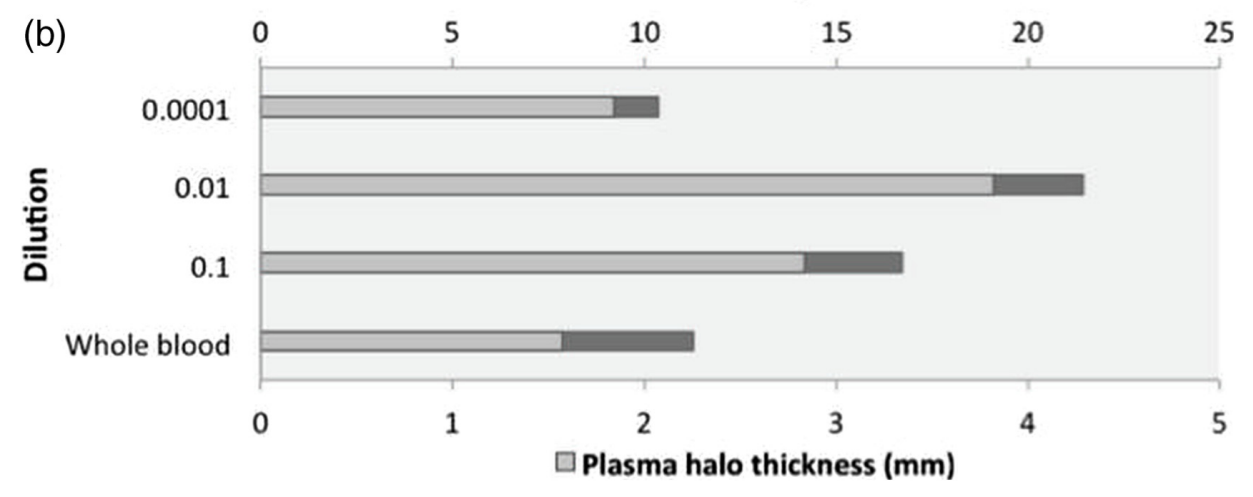

Fig. 7 CAFA with whole blood and different blood dilutions. (a) Plasma separation in five spots corresponding to different blood dilutions: 1 . Whole blood; 2 . Dilution $10^{-1} ; 3$. Dilution $10^{-2} ; 4$. Dilution $10^{-4} ; 5$. Water control. (b) Measurements of intensity and thickness of plasma halo for samples: whole blood, dilution $10^{-1}$, dilution $10^{-2}$, and dilution $10^{-4}$. 
in the edges of the spot, and little or no signal is observed in the center. Depending on the porosity of the membrane, the red blood cells either are retained or let through by the membrane. ${ }^{45,46}$ The red blood cells have an average size of $5 \mu \mathrm{m} ;{ }^{46}$ therefore, it can be assumed that the red cells are retained on the membrane, whereas the plasma migrates by capillarity through the pores. The center of the spot has no fluorescence because the chimeric probe does not bind to red cells, which is why fluorescence is observed only in the plasma, where thrombin is concentrated.

An essential factor to consider is the dilution of the blood used. The sensitivity of the assay depends directly on the volume of plasma separated from the blood sample. ${ }^{47}$ When a sample of whole blood is used, the plasma does not separate successfully; this may be because the red cells are very concentrated and clog the membrane. When the blood sample is diluted, the membrane is not saturated, and plasma separation occurs efficiently. As a measure of the efficiency of the separation, we evaluated the thickness and intensity of the plasma halo obtained. It is observed that at a dilution of $10^{-2}$ the plasma halo is thicker and more intense [Fig. 7(b)]. Therefore, a $10^{-2}$ dilution of blood that was found to be optimal to carry out the test of the CAFA is proposed here.

Finally, we investigated whether the blood used should be treated or not with an anticoagulant. For this, we compared the intensity of the signal and the plasma halo thickness obtained in the presence and absence of citrate. The results in both cases did not give significant differences (Fig. 8). Therefore, for this test, we conclude that the use of anticoagulant agents is not necessary.

(a) Without anticoagulant
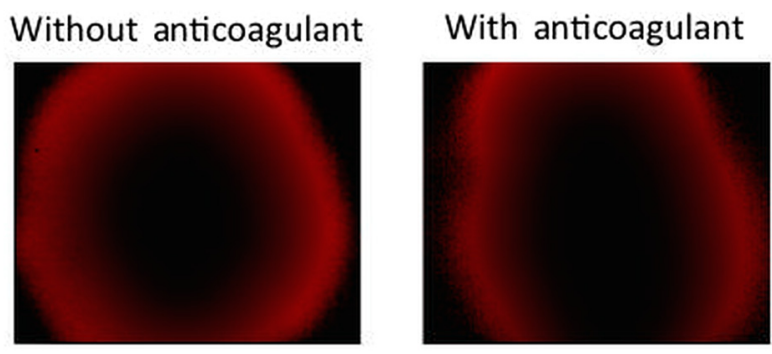

(b)

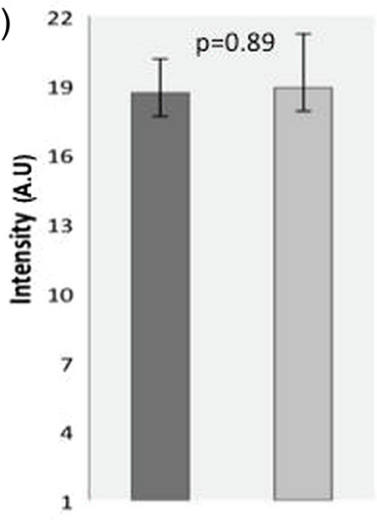

$\square$ With anticoagulant

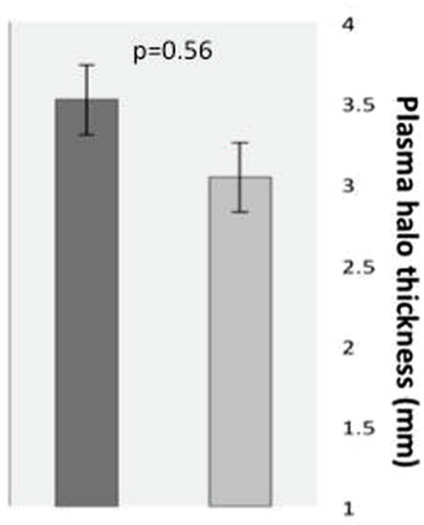

Without anticoagulant

Fig. 8 Comparison between blood samples extracted with and without citrate as anticoagulant. (a) Optical microscopy images of the CAFA results and (b) measurements of intensity and thickness of plasma separation halo for blood samples extracted with and without citrate. To compare between assays, an ANOVA one-way was performed, and the $p$-values are shown in the figure.
In this way, with the use of direct blood samples, the biosensor we introduced here meets the requirements to be used as a POC device. ${ }^{6,48,49}$ In support of this, we have that it works with: (i) low-volume clinical samples, (ii) minimum user manipulation, (iii) inexpensive disposable membrane for blood separation, and (iv) low-cost equipment detection of proteins.

\subsection{Chimeric Aptafluorescence Assay Strategy Advantages}

The synergistic use of the labeled-free CAFA strategy presented here shows a promising alternative in comparison with more involved techniques used for protein detection. The use of NMM IX dye with the parallel G-quadruplex DNA made this assay very sensitive, reliable, and easily adaptable to detect other biological targets.

The results obtained with the CAFA are comparable, and in some cases, better than those obtained with other reported techniques (Table 2). Although some authors report lower detection limits than those obtained in this work, most of these studies employ detection techniques that require highly sophisticated equipment or expensive reagents, such as labeled oligonucleotides. ${ }^{50-56}$ Additionally, none of the other reported techniques in Table 2 employ whole blood as a starting sample, or uses a chimeric label-free aptamer. For the application assay we only required an oligonucleotide, simple folding conditions plus a dye that is noncovalent bound to the DNA, which guarantees the low cost of the system in comparison with labeled probes. ${ }^{57}$ The qualities of being inexpensive and straightforward for our detection technique and further that we can use whole nonseparated blood are extremely useful for the development of a POC device.

In this manner, the main advantages of our aptamer strategy are six: (i) it demands minimal amounts of reagents (aliquots of $2 \mu \mathrm{L}$ or less with $\mu \mathrm{M}$ concentrations), (ii) nucleic acid optimized aptamers are significantly more resistant than antibodies to denaturation and can be refolded if needed, (iii) aptamer at

Table 2 Comparison of different methods for thrombin detection.

\begin{tabular}{|c|c|c|c|}
\hline References & Detection method & $\begin{array}{l}\text { Detection } \\
\text { limit }\end{array}$ & $\begin{array}{l}\text { Labeled } \\
\text { probe }\end{array}$ \\
\hline Cho et al. ${ }^{50}$ & Raman spectroscopy & $100 \mathrm{pM}$ & Yes \\
\hline Lin et al. ${ }^{51}$ & Guided-mode resonance & $0.19 \mu \mathrm{M}$ & No \\
\hline Jiang et al. ${ }^{52}$ & Chemiluminescence & $80 \mathrm{pM}$ & Yes \\
\hline Coelho et al. ${ }^{53}$ & $\begin{array}{l}\text { Optical long period fiber } \\
\text { grating sensors and surface } \\
\text { plasmon resonance }\end{array}$ & $10 \mathrm{nM}$ & Yes \\
\hline Polonschii et al. ${ }^{54}$ & Surface plasmon resonance & $2.5 \mathrm{nM}$ & Yes \\
\hline Lim et al. ${ }^{55}$ & $\begin{array}{l}\text { Electrochemical impedance } \\
\text { spectroscopy }\end{array}$ & $2.7 \mu \mathrm{M}$ & No \\
\hline Yang et al. ${ }^{56}$ & $\begin{array}{l}\text { Cyclic voltammetry and } \\
\text { differential pulse voltammetry }\end{array}$ & $5.6 \mathrm{pM}$ & No \\
\hline
\end{tabular}

$\begin{array}{lll}\text { This work } & \text { Colorimetric quantification } & 0.11 \mu \mathrm{M} \quad \text { No }\end{array}$ 
the conditions reported here are cost competitive with antibodies, (iv) multiple samples can be measured simultaneously as digital imaging is used for acquisition and image processing/quantification could be automated and finally, (v) the one-step methodology introduced here is fast and straightforward to apply.

\section{Conclusions}

We have developed a fast and easy-to-use aptasensor and demonstrated its capabilities to detect proteins of biological and physiological interest in blood samples. Sensibility, linearity, and LOD are perfect for a low cost and rapid assay based on a label-free strategy. The CAFA works by implementing two modules: the first, a detection module — aptamer — and second, a transduction module-sequence that induces specific fluorescence of NMM IX. Most importantly, this design can be adaptable to other analytes that are either proteins or other metabolites in blood, by changing the specific sequence of the detection module. We have also demonstrated that the biosensor is amenable to be used as a POC device.

\section{Disclosures}

Based on the International Committee of Medical Journal Editors recommendations, the authors have nothing to disclose.

\section{Acknowledgments}

This work was supported by the program USFQ Chancellor Grants 2015. We thank the Molecular Biology Laboratories from the School of Medicine at Universidad San Francisco de Quito for the resources provided and for all the support given to fulfill this project. We also thank the HPC-USFQ for the facilities for data storage and computer resources.

\section{References}

1. G. A. Zelada-Guillén et al., "Ultrasensitive and real-time detection of proteins in blood using a potentiometric carbon-nanotube aptasensor," Biosens. Bioelectron. 41, 366-371 (2013).

2. S. Song, H. Xu, and C. Fan, "Potential diagnostic applications of biosensors: current and future directions," Int. J. Nanomedicine 1, 433-440 (2006).

3. J. E. Hale, "Advantageous uses of mass spectrometry for the quantification of proteins," Int. J. Proteomics 2013, 1-6 (2013).

4. B. Piro et al., "Comparison of electrochemical immunosensors and aptasensors for detection of small organic molecules in environment, food safety, clinical and public security," Biosensors 6, 7 (2016).

5. E. van Duijn, "Current limitations in native mass spectrometry based structural biology," J. Am. Soc. Mass Spectrom. 21, 971-978 (2010).

6. V. Gubala et al., "Point of care diagnostics: status and future," Anal. Chem. 84, 487-515 (2012).

7. A. Warsinke, "Point-of-care testing of proteins," Anal. Bioanal. Chem. 393, 1393-1405 (2009).

8. A. B. Dobrovolsky et al., "Inhibition of thrombin activity with DNAaptamers," Bull. Exp. Biol. Med. 148, 33-36 (2009).

9. R. Nezlin, "Use of aptamers in immunoassays," Mol. Immunol. 70, 149154 (2016).

10. R. Nutiu and Y. Li, "In vitro selection of structure-switching signaling aptamers," Angew. Chem. 117, 1085-1089 (2005).

11. A. Sassolas, L. J. Blum, and B. D. Leca-Bouvier, "Immobilization strategies to develop enzymatic biosensors," Biotechnol. Adv. 30, 489-511 (2012).

12. C.-C. Huang et al., "Aptamer-functionalized gold nanoparticles for turnon light switch detection of platelet-derived growth factor," Anal. Chem. 79, 4798-4804 (2007).

13. Z. Zhu et al., "Label-free aptamer-based sensors for L-argininamide by using nucleic acid minor groove binding dyes," Chem. Commun. 47, 3192-3194 (2011).
14. X. Chen et al., "A label-free fluorescence strategy for selective detection of nicotinamide adenine dinucleotide based on a dumbbell-like probe with low background noise," Biosens. Bioelectron. 77, 486-490 (2016).

15. K. Wang et al., "A label-free aptasensor for highly sensitive detection of ATP and thrombin based on metal-enhanced PicoGreen fluorescence," Biosens. Bioelectron. 63, 172-177 (2015).

16. N. C. Sabharwal et al., "N-methylmesoporphyrin IX fluorescence as a reporter of strand orientation in guanine quadruplexes," FEBS $J$. 281, 1726-1737 (2014).

17. L. Guo et al., "A G-quadruplex based label-free fluorescent biosensor for lead ion," Biosens. Bioelectron. 35, 123-127 (2012).

18. Y. Guo et al., "A label-free biosensor for DNA detection based on ligand-responsive G-quadruplex formation," Talanta 114, 138-142 (2013).

19. Y. He and B. Jiao, "Simple and convenient G-quadruplex-based fluorescence assay of micrococcal nuclease activity," $R S C A d v .5,104116-$ 104121 (2015).

20. D.-L. Ma et al., "Recent developments in G-Quadruplex probes," Chem. Biol. 22, 812-828 (2015).

21. B. Deng et al., "Aptamer binding assays for proteins: the thrombin example-a review," Anal. Chim. Acta 837, 1-15 (2014).

22. D. M. Tasset, M. F. Kubik, and W. Steiner, "Oligonucleotide inhibitors of human thrombin that bind distinct epitopes," J. Mol. Biol. 272, 688698 (1997).

23. K. E. Brummel-Ziedins et al., "Thrombin generation profiles in deep venous thrombosis," J. Thromb. Haemost. 3, 2497-2505 (2005).

24. M. A. Shuman and P. W. Majerus, "The measurement of thrombin in clotting blood by radioimmunoassay," J. Clin. Invest. 58, 1249-1258 (1976).

25. M. A. Mendez and V. A. Szalai, "Synapsable quadruplex-mediated fibers," Nanoscale Res. Lett. 8, 210 (2013).

26. S. E. Evans et al., "End-stacking of copper cationic porphyrins on parallel-stranded guanine quadruplexes," JBIC J. Biol. Inorg. Chem. 12, 1235-1249 (2007).

27. A. V. Cherepanova et al., "Blood deoxyribonuclease activity in health and diseases," Biomed. Chem. 1, 299-304 (2007).

28. D. Suck, "DNA recognition by DNase I," J. Mol. Recognit. 7, 65-70 (1994).

29. A. Lahm and D. Suck, "DNase I-induced DNA conformation. 2 A structure of a DNase I-octamer complex,' J. Mol. Biol. 222, 645-667 (1991).

30. W. A. Al-Soud and P. Rådström, "Purification and characterization of PCR-inhibitory components in blood cells," J. Clin. Microbiol. 39, 485-493 (2001).

31. J. He and P. Furmanski, "Sequence specificity and transcriptional activation in the binding of lactoferrin to DNA," Nature 373, 721-724 (1995).

32. C. A. Schneider, W. S. Rasband, and K. W. Eliceiri, "NIH Image to ImageJ: 25 years of image analysis," Nat. Methods 9, 671-675 (2012).

33. W. F. DeGrado, "Computational biology: biosensor design," Nature 423, 132-133 (2003).

34. R. Penchovsky, "Computational design and biosensor applications of small molecule-sensing allosteric ribozymes," Biomacromolecules 14, 1240-1249 (2013).

35. V. M. Marathias et al., "Determination of the number and location of the manganese binding sites of DNA quadruplexes in solution by EPR and NMR in the presence and absence of thrombin," J. Mol. Biol. 260, 378394 (1996).

36. K. Padmanabhan and A. Tulinsky, "An ambiguous structure of a DNA 15-mer thrombin complex," Acta Crystallogr. D Biol. Crystallogr. 52, 272-282 (1996).

37. I. R. Krauss et al., "High-resolution structures of two complexes between thrombin and thrombin-binding aptamer shed light on the role of cations in the aptamer inhibitory activity," Nucleic Acids Res. 40, 8119-8128 (2012).

38. A. Ambrus et al., "Solution structure of the biologically relevant G-quadruplex element in the human c-MYC promoter. Implications for G-quadruplex stabilization,” Biochemistry 44, 2048-2058 (2005).

39. R. I. Mathad et al., "c-MYC promoter G-quadruplex formed at the 5'-end of NHE III1 element: insights into biological relevance and parallel-stranded G-quadruplex stability," Nucleic Acids Res. 39, 90239033 (2011). 
40. A. T. Phan et al., "Structure of an unprecedented G-quadruplex scaffold in the human c-kit promoter," J. Am. Chem. Soc. 129, 4386-4392 (2007).

41. J. M. Nicoludis et al., "Interaction of human telomeric DNA with N-methyl mesoporphyrin IX," Nucleic Acids Res. 40, 5432-5447 (2012).

42. J. M. Nicoludis et al., "Optimized end-stacking provides specificity of N-methyl mesoporphyrin IX for human telomeric G-quadruplex DNA," J. Am. Chem. Soc. 134, 20446-20456 (2012).

43. J. N. Miller and J. C. Miller, Statistics and Chemometrics for Analytical Chemistry, Prentice Hall, Englewood Cliffs, New Jersey (2000).

44. M. Xiao et al., "Analysis of the whole serum proteome using an integrated 2D LC-MS/MS system," Anal. Methods 6, 7157-7160 (2014).

45. M. A. Mansfield, "The use of nitrocellulose membranes in lateral-flow assays," in Drugs of Abuse, R. C. Wong and H.Y. Tse, Eds., pp. 71-85, Humana Press, New York (2005).

46. LXN Corporation, "Whole blood separation method and devices using the same," US5725774A (1995).

47. C. A. Holstein et al., "Immobilizing affinity proteins to nitrocellulose: a toolbox for paper-based assay developers," Anal. Bioanal. Chem. 408, 1335-1346 (2016).

48. M. Sajid, A.-N. Kawde, and M. Daud, "Designs, formats and applications of lateral flow assay: a literature review," J. Saudi Chem. Soc. 19, 689-705 (2015).

49. A. K. Yetisen, M. S. Akram, and C. R. Lowe, "Paper-based microfluidic point-of-care diagnostic devices," Lab Chip 13, 2210-2251 (2013).

50. H. Cho et al., "Aptamer-based SERRS sensor for thrombin detection," Nano Lett. 8, 4386-4390 (2008).

51. S.-F. Lin et al., "A guided mode resonance aptasensor for thrombin detection," Sensors 11, 8953-8965 (2011).

52. Z. Jiang et al., "An aptamer-based biosensor for sensitive thrombin detection with phthalocyanine@ $\mathrm{SiO}_{2}$ mesoporous nanoparticles," Biosens. Bioelectron. 53, 340-345 (2014).

53. L. Coelho et al., "Aptamer-based fiber sensor for thrombin detection," J. Biomed. Opt. 21, 087005 (2016).

54. C. Polonschii et al., "A novel low-cost and easy to develop functionalization platform. Case study: aptamer-based detection of thrombin by surface plasmon resonance," Talanta 80, 2157-2164 (2010).

55. T. Lim et al., "Microfluidic biochips for simple impedimetric detection of thrombin based on label-free DNA aptamers," BioChip J. 11, 109-115 (2017).
56. J. Yang et al., "Proximity binding and metal ion-dependent DNAzyme cyclic amplification-integrated aptasensor for label-free and sensitive electrochemical detection of thrombin," Anal. Chem. 88, 8218-8223 (2016).

57. A. M. Montero, "Computer aided design of aptamer for prothrombin detection in blood," Biophys. J. 108(2), 483a (2015)

Andrea Montero-Oleas is a biotechnological processes engineer at Universidad San Francisco de Quito, Ecuador, and received her MSc degree in biomedicine from the Universidad de Castilla la Mancha, Spain. Currently, she works as professor in the biotechnology department at Universidad San Francisco de Quito. Her research interests are related to molecular virology and the development of nanosensors based on DNA as a building material with agronomic, medical, and microbiological interest.

Cesar Costa-Vera is a professor in the Department of Physics of the Escuela Politécnica Nacional in Quito. He is the leader of the Mass Spectrometry and Optical Spectroscopy Group (MSOS). He is a member of the Academy of Sciences of Ecuador. His research interests are related the development of methods and instrumentation in optics, laser applications, photonics, and nanospectroscopy. He has worked in applications to forensic, patrimonial, industrial, environmental and geophysical, and medical and biological problems.

Elizabeth Samaniego Onofre is a soon-to-graduate physicist with an interest in pursuing a career in biomedicine. Presently, she is working on the application of optical, noninvasive methods to quantify biosensors based on DNA nonconventional structures. She has also worked on the polymer modeling for hydrogel synthesis and biosensors.

Miguel Angel Mendez obtained his PhD at University of Maryland, Baltimore County. Currently, he works at the Department of Chemical Engineering at Universidad San Francisco de Quito. His past research projects concentrated in nanotechnology and material sciences. He worked with DNA as a novel material for electronics and organizational nanoscaffold. His current work focuses on the development of detection strategies/imaging for biomedical and basic science applications based on DNA materials. 